\title{
MicroRNA-506-3p targets SIRT1 and suppresses AMPK pathway activation to promote hepatic steatosis
}

\author{
LIANG-KAI HU $^{1 *}$, JIAN-QING CHEN ${ }^{1 *}$, HAO ZHENG $^{2-4 *}$, \\ YUAN-PING TAO ${ }^{2-4^{*}}$, YUAN YANG ${ }^{2-4}$ and XUAN-FU XU ${ }^{1}$ \\ ${ }^{1}$ Department of Gastroenterology, Shidong Hospital; ${ }^{2}$ Third Department of Hepatic Surgery, \\ Eastern Hepatobiliary Surgery Hospital, Second Military Medical University; ${ }^{3}$ Key Laboratory of \\ Signaling Regulation and Targeting Therapy of Liver Cancer (SMMU), Ministry of Education; \\ ${ }^{4}$ Shanghai Key Laboratory of Hepatobiliary Tumor Biology (EHBH), Shanghai 200438, P.R. China
}

Received December 9, 2019; Accepted March 18, 2021

DOI: 10.3892/etm.2021.10865

\begin{abstract}
Nonalcoholic fatty liver disease (NAFLD) is a complex type of liver disease that represents an important global health threat. The mechanistic basis of this disease remains incompletely understood. The present study sought to explore whether microRNA (miR)-506-3p served a functional role in the onset and/or progression of NAFLD. To that end, high levels of glucose were used to treat liver cancer cell lines (HepG2 and Huh7) to model hepatic steatosis, and the expression levels of miR-506-3p and its downstream target genes were assessed. The cells of this hepatic steatosis model were transfected with miR-506-3p mimic molecules to explore the effect of miR-506-3p overexpression on cell viability, target gene expression and AMP-activated protein kinase (AMPK) phosphorylation. Via bioinformatics approaches, sirtuin 1 (SIRT1) was identified as a potential miR-506-3p target gene with relevance in NAFLD, and this interaction was confirmed via luciferase reporter assay. In the hepatic steatosis model of the present study, miR-506-3p expression level was significantly increased, whereas SIRT1 mRNA/protein levels and AMPK phosphorylation levels were markedly decreased. Transfection of the cells with miR-506-3p mimics led to significant SIRT1 downregulation, while miR-506-3p inhibitor molecules exhibited the opposite effect, with similar trends observed in the phosphorylation status of AMPK. These results suggested that miR-506-3p can inhibit SIRT1 expression and associated AMPK phosphorylation in HepG2 and Huh7 cells in an in vitro hepatic steatosis model system. These data indicated
\end{abstract}

Correspondence to: Professor Xuan-Fu Xu, Department of Gastroenterology, Shidong Hospital, 999 Shiguang Road, Yangpu, Shanghai 200438, P.R. China

E-mail: shuanfusky@163.com

${ }^{*}$ Contributed equally

Key words: AMP-activated protein kinase, liver, microRNA506-3p, nonalcoholic fatty liver disease, sirtuin 1 that the miR-506-3p/SIRT1/AMPK axis may be valuable as a therapeutic target in patients affected by NAFLD.

\section{Introduction}

Obesity is a metabolic disorder that remains a major global public health threat, with affected individuals frequently suffering from associated conditions, including type 2 diabetes and nonalcoholic fatty liver disease (NAFLD) (1). The incidence rate of fatty liver was $50 \%$ in patients with type 2 diabetes patients and incidence rate of fatty liver in alcoholics was $58 \%$ (1). In NAFLD, lipids accumulate at abnormal levels within hepatic cells and drive significant inflammatory activity, oxidative stress, fibrosis, metabolic dysregulation and liver cancer development (1,2). Although a wide range of metabolic and genetic factors have been revealed to contribute to the development and progression of NAFLD, the etiology of this disease remains incompletely understood (2).

A previous study has suggested that the NAD-dependent deacetylase sirtuin 1 (SIRT1), which is a metabolic homeostasis regulator, may be associated with the development of NAFLD (3). High levels of SIRT1 expression were found in the NALF liver, which has been indicated to result in a notable upregulation of genes associated with lipogenesis and gluconeogenesis compared to that in the normal/healthy liver, thereby leading to an important increase in intracellular glucose and lipid concentrations $(3,4)$. Consistent with this model, individuals with obesity suffering from diabetes and NAFLD have been indicated to express lower SIRT1 levels compared with those in healthy controls $(5,6)$.

The serine threonine kinase liver kinase B1 (LKB1) has been demonstrated to phosphorylate and activate AMP-activated protein kinase (AMPK), which regulates the metabolism of lipids and carbohydrates within cells and detects their energy status (7). Importantly, SIRT1 is known to activate LKB1 by deacetylation, leading to AMPK activation (8). The activation of AMPK alters the $\mathrm{NAD}^{+} / \mathrm{NADH}$ ratio in cells, resulting in increased SIRT1 activity in a metabolic regulatory feedback mechanism (8). Short non-coding microRNA (miRNA/miR) molecules have been indicated to be highly conserved molecular entities capable of binding to the 3'-untranslated region 
(3'-UTR) of specific target mRNA molecules, resulting in a significant suppression of target gene expression either via translational repression or mRNA cleavage $(9,10)$. Since miRNAs are important in all physiological contexts, the relevance of miRNA dysregulation in metabolic disease contexts has been specifically highlighted (11-14). A previous study has suggested that miR-506-3p dysregulation may be associated with the onset and progression of multiple forms of cancer (15). Previous studies have indicated that miR-506-3p may suppress hepatocellular carcinoma (HCC) metastasis $(16,17)$. Given that NAFLD is an important cause of HCC (18) and considering the close association between miR-506-3p and HCC, the present study aimed to uncover the role of miR-506-3p in NAFLD. Subsequently, the association of miR-506-3p with the pathogenesis of NAFLD was explored, in order to study the underlying molecular mechanisms of this condition.

\section{Materials and methods}

Cell culture and treatment. HepG2 and Huh7 cell lines were obtained from the American Type Culture Collection and analyzed with short tandem repeat profiling semiannually after the first recovery. HepG2 and Huh7 cells were cultured in DMEM (Gibco; Thermo Fisher Scientific, Inc.) containing 10\% FBS (HyClone; Cytiva), D-glucose (5.5 mM) and 1\% penicillin/streptomycin at $37^{\circ} \mathrm{C}$ in a $5 \% \mathrm{CO} 2$ incubator. To model hepatic steatosis, the cells were plated in 6-well plates overnight, followed by a 4-h treatment in serum-free DMEM that was subsequently supplemented with $100 \mathrm{mM}$ D-glucose for an additional $48 \mathrm{~h}$ at $37^{\circ} \mathrm{C}$ and the control group was treated with DMSO.

Oil Red $O$ staining. HepG 2 and Huh7 cells $\left(1 \times 10^{6}\right.$ cells/per well) were seeded into 24 -well plates, $4 \%$ formalin-fixed at room temperature for $1 \mathrm{~h}$ and subsequently stained for $15 \mathrm{~min}$ at room temperature with Oil Red $\mathrm{O}$ solution (cat. no. ab223796; Abcam) at room temperature to detect intracellular lipids upon microscopic analysis. In addition, Oil Red $\mathrm{O}$ staining quantification was performed by treating samples with isopropanol to solubilize the dye, and the absorbance of the isopropanol solution was subsequently quantified at $510 \mathrm{~nm}$ with a microplate reader.

Intracellular triglyceride (TG) measurements. TG levels were measured as in a previous study (19). HepG2 and Huh7 cells $\left(1 \times 10^{6}\right.$ cells/per well) were seeded into 24 -well plates and were lysed in NP-40 for $1 \mathrm{~h}$ at room temperature. Subsequently, lysates were warmed for $5 \mathrm{~min}$ to $100^{\circ} \mathrm{C}$ and cooled to room temperature. This procedure was repeated one additional time to fully solubilize lipids within these samples. Samples were then centrifuged, and TG levels were measured using an enzymatic TG assay kit (cat. no. K614-100; Biovision, Inc.) according to the manufacturer's protocols, with total protein levels in each sample used to normalize the TG content.

miRNA transfection. miR-506-3p mimics (50 nM), inhibitor $(50 \mathrm{nM})$ and corresponding controls $(50 \mathrm{nM})$ were purchased from Shanghai GenePharma Co., Ltd. Polyethylenimine (PEI; Sigma-Aldrich; Merck KGaA) was used to transfect all cells
Table I. Sequences of primers used for reverse transcriptionquantitative PCR.

\begin{tabular}{ll}
\hline Primer & \multicolumn{1}{c}{ Sequence (5'-3') } \\
\hline SIRT1 & F: TGCGGGAATCCAAAGGATAA \\
& R: CAGGCAAGATGCTGTTGCA \\
miR-506-3p & F: TAAGGCACCCTTCTGAGTAGA \\
& R: GCGAGCACAGAATTAATACGAC \\
SREBP1 & F: CCACAATGCCATTGAGAAGCG \\
& R: CTGACACCAGGTCCTTCAGTG \\
FASN & F: TTGCTGGCACTACAGAATGC \\
& R: AACAGCCTCAGAGCGACAAT \\
SCD1 & F: CACACCTTCCCCTTCGACTA \\
& R: TGACTCCCGTCTCCAGTTCT \\
ACC1 & F: CTTGGAGCAGAGAACCTTCG \\
& R: ACTTCCCGACCAAGGACTTT \\
U6 & F: CTCGCTTCGGCAGCACA \\
& R: AACGCTTCACGAATTGCGT \\
GAPDH & F: TGTGGGCATCAATGGATTTGG \\
& R: ACACCATGTATTCCGGGTCAAT
\end{tabular}

SIRT1, sirtuin 1; miR, microRNA; SREBP1, sterol regulatory element-binding protein 1; FASN, fatty acid synthase; SCD1, stearoyl-CoA desaturase-1; ACC1, acetyl-CoA carboxylase 1; F, forward; $\mathrm{R}$, reverse.

in the present study. For transfection, $1 \times 10^{5}$ HepG2 and Huh7 cells were plated for $24 \mathrm{~h}$ at $37^{\circ} \mathrm{C}$ in serum- and antibioticfree DMEM, following which PEI-miRNA complexes in Opti-MEM (Gibco; Thermo Fisher Scientific, Inc.) were added. After $4 \mathrm{~h}$, fresh DMEM containing 10\% FBS was added, and the cells were incubated for an additional $48 \mathrm{~h}$. miR505-3p mimics (5'-GGGAGCCAGGAACGAUUGAUGU-3'), inhibitor (5'-ACUACUGAGCCGCAGUAGA-3'), mimics-NC (5'-UUCUCCGAACGUGUCACGUTT-3') and inhibitor-NC (5'-UUCUCCGAACGUGUCACGUTT-3') were used.

$R N A$ isolation and reverse transcription quantitative- $P C R$ (RT-qPCR). Total RNA was extracted from HepG2 and Huh7 cells using TRIzol ${ }^{\circledR}$ Reagent (Thermo Fisher Scientific, Inc.) according to the manufacturer's protocols. Total RNA was reverse transcribed into cDNA using the HiFiScipt cDNA Synthesis kit (CoWin Biosciences) at $16^{\circ} \mathrm{C}$ for $30 \mathrm{~min}, 42^{\circ} \mathrm{C}$ for $30 \mathrm{~min}$ and $85^{\circ} \mathrm{C}$ for $5 \mathrm{~min}$. qPCR was performed using SYBR $^{\circledR}$ Premix Ex Taq ${ }^{\mathrm{TM}}$ (Takara Biotechnology Co., Ltd.) and the ABI PRISM 7500 real-time PCR system (Applied Biosystems; Thermo Fisher Scientific, Inc.) according to the manufacturer's protocol. qPCR reaction was performed using the following conditions: Pre-denaturation at $95^{\circ} \mathrm{C}$ for $1 \mathrm{~min}$, 40 cycles of denaturation at $95^{\circ} \mathrm{C}$ for $30 \mathrm{sec}$, annealing at $67^{\circ} \mathrm{C}$ for $30 \mathrm{sec}$ and extension at $72^{\circ} \mathrm{C}$ for $30 \mathrm{sec}$, followed by a final extension step at $72^{\circ} \mathrm{C}$ for $5 \mathrm{~min}$. The sequences of the RT-qPCR primers were listed in Table I. miRNA and mRNA expression levels were quantified using the $2^{-\Delta \Delta C q}$ method (20) and normalized to the internal reference genes U6 and GAPDH, respectively. 
A

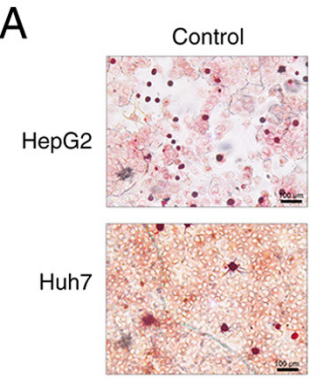

Treated

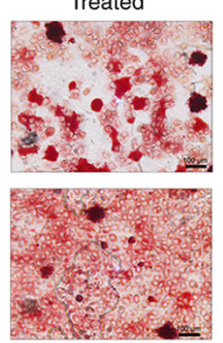

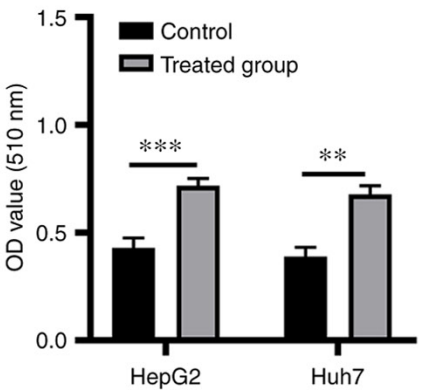
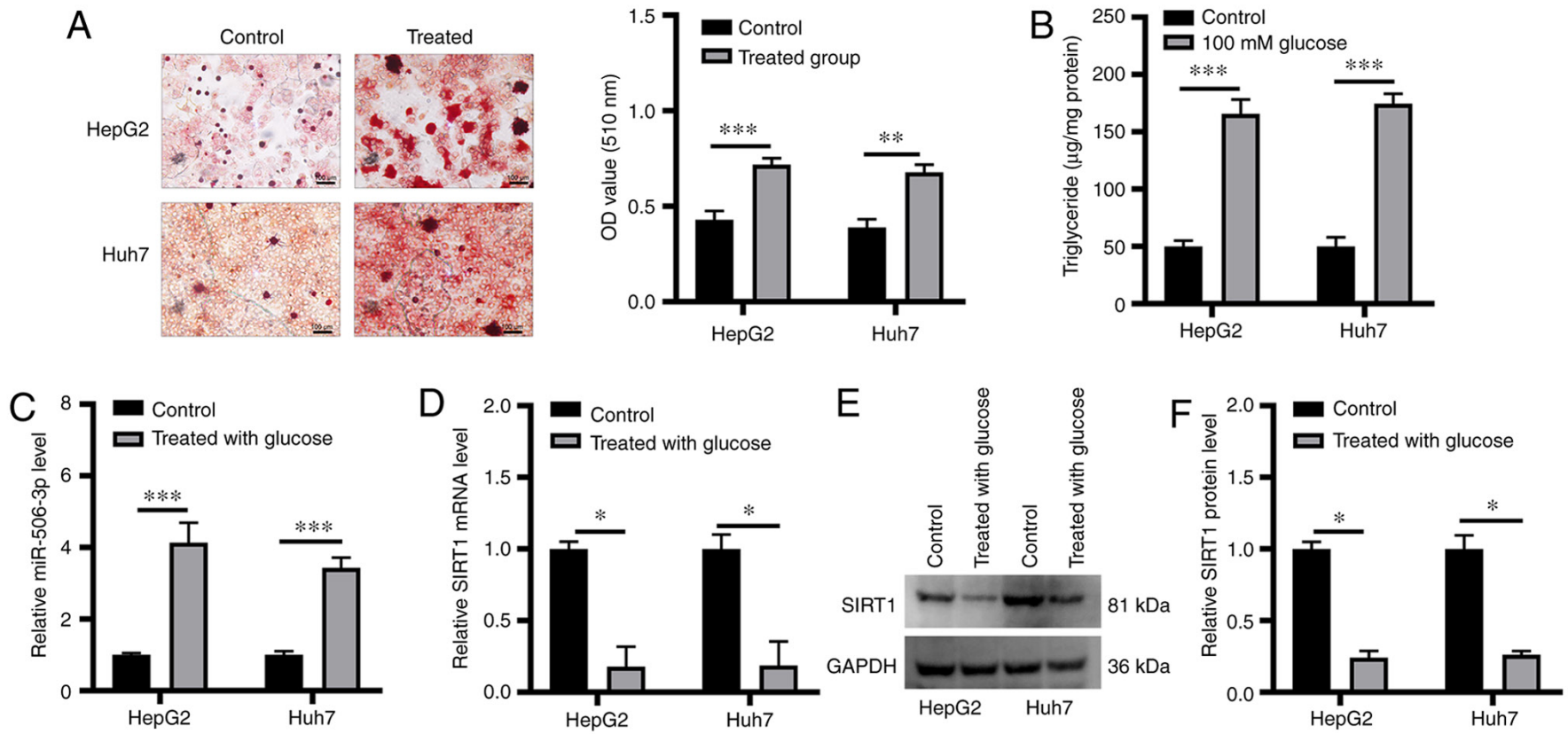

Figure 1. miR-506-3p and SIRT1 expression is altered in a model of hepatic steatosis. (A) Left panel: Oil Red O staining of HepG2 and Huh7 cells after incubation with $100 \mathrm{mM}$ glucose. Right panel: Quantification via spectrophotometry. Scale bars, $100 \mu \mathrm{m}$. (B) Triglyceride levels were assessed in HepG2 and Huh7 cells after incubation with $100 \mathrm{mM}$ glucose and control cells. Reverse transcription-quantitative PCR was used to measure (C) miR-506-3p and (D) SIRT1 mRNA levels in high-glucose treated cells. (E) SIRT1 protein levels were assessed in these cells via western blotting, (F) with densitometric quantification performed using ImageJ. Data are presented as the mean $\pm \mathrm{SD}(\mathrm{n}=3)$. ${ }^{*} \mathrm{P}<0.05 ;{ }^{* *} \mathrm{P}<0.01 ;{ }^{* * *} \mathrm{P}<0.001$. miR, microRNA; SIRT1, sirtuin 1 ; OD, optical density.

Western blotting. Huh7 and HepG2 cells were harvested and lysed in RIPA lysis buffer supplemented with protease inhibitors (Bio Basic Inc.). A bicinchoninic acid protein assay kit (Thermo Fisher Scientific, Inc.) was used to quantify protein levels in each sample, following which $30 \mu \mathrm{g}$ protein/sample were separated via $10 \%$ SDS-PAGE prior to transfer onto PVDF membranes (Millipore Sigma). The blots were then blocked using either 5\% BSA (Gibco; Thermo Fisher Scientific, Inc.) for AMKP and phosphorylated (p)-AMPK or non-fat milk in TBST (for other proteins), followed by overnight incubation with antibodies specific to SIRT1 (1:1,000; cat. no. 8469), AMPK (1:1,000; cat. no. 5831), p-AMPK (Threonine 172; 1:1,000; cat. no. 8208) and GAPDH (1:1,000; cat. no. 5174; all from Cell Signaling Technology, Inc.) at $4^{\circ} \mathrm{C}$. The blots were subsequently probed for $1 \mathrm{~h}$ at $4^{\circ} \mathrm{C}$ with an appropriate horseradish peroxidase-linked rabbit (1:10,000; cat. no. 7074) and anti-mouse IgG (1:10,000; cat. no. 7076; Cell Signaling Technology, Inc.) secondary antibodies followed by enhanced chemiluminescent substrate visualization (GE Healthcare). ImageJ Software version 1.46 (National Institutes of Health) was used for densitometric analyses.

SIRT1 activity measurement. SIRT1 activity in HepG2 and Huh7 cells transfected with either miR-506-3p mimics or inhibitor was quantified with a SIRT1 fluorometric assay kit (cat. no. CS1040; Sigma-Aldrich; Merck KGaA) using manufacturer's protocols. For this assay, a substrate that contained both a fluorophore and a quencher was used, so that following SIRT1-mediated deacetylation in the presence of NAD, this substrate was cleaved by a peptidase, leading to a fluorescent signal proportional to the degree of SIRT1 activity. Excitation and emission wavelengths at 350 and $460 \mathrm{~nm}$, respectively, were applied. Recombinant SIRT1 and fluoro-deacetylated peptides were used as positive controls. NAD, samples or enzymes were omitted from the assays as negative controls.

Luciferase reporter assay. The wild-type or mutated versions of SIRT1 3'-UTR were cloned downstream of the firefly gene in the psiCHECK2 plasmid (Promega Corporation), with the mutant construct containing mutations designed to disrupt miR-506-3p binding to its cognate target sequence. PEI transfection reagent (Sigma-Aldrich; Merck KGaA) was used to transfect HepG2 and Huh7 cells with either miR-506-3p mimics or inhibitor together with the vectors encompassing the wild-type or mutated versions of SIRT1 3'-UTR. Luciferase activity in these cells was analyzed at $48 \mathrm{~h}$ post-transfection using a Dual-Luciferase Reporter Assay System (Promega Corporation) based on the manufacturer's instructions, with Renilla activity used for normalization purposes.

Statistical analysis. GraphPad Prism 6 (GraphPad Software, Inc.) was used for all statistical analyses in the present study. Data are presented as the mean \pm standard deviation from three experimental repeats and one-way ANOVA followed by Tukey's post hoc test was used to compare different groups. $\mathrm{P}<0.05$ was considered to indicate a statistically significant difference.

\section{Results}

miR-506-3p and SIRT1 expression is altered in a model of hepatic steatosis. Huh7 and HepG2 cells treated with high glucose concentration were used to model hepatic steatosis. Lipid accumulation within these cells was confirmed via Oil Red O staining (Fig. 1A). TG content was significantly 

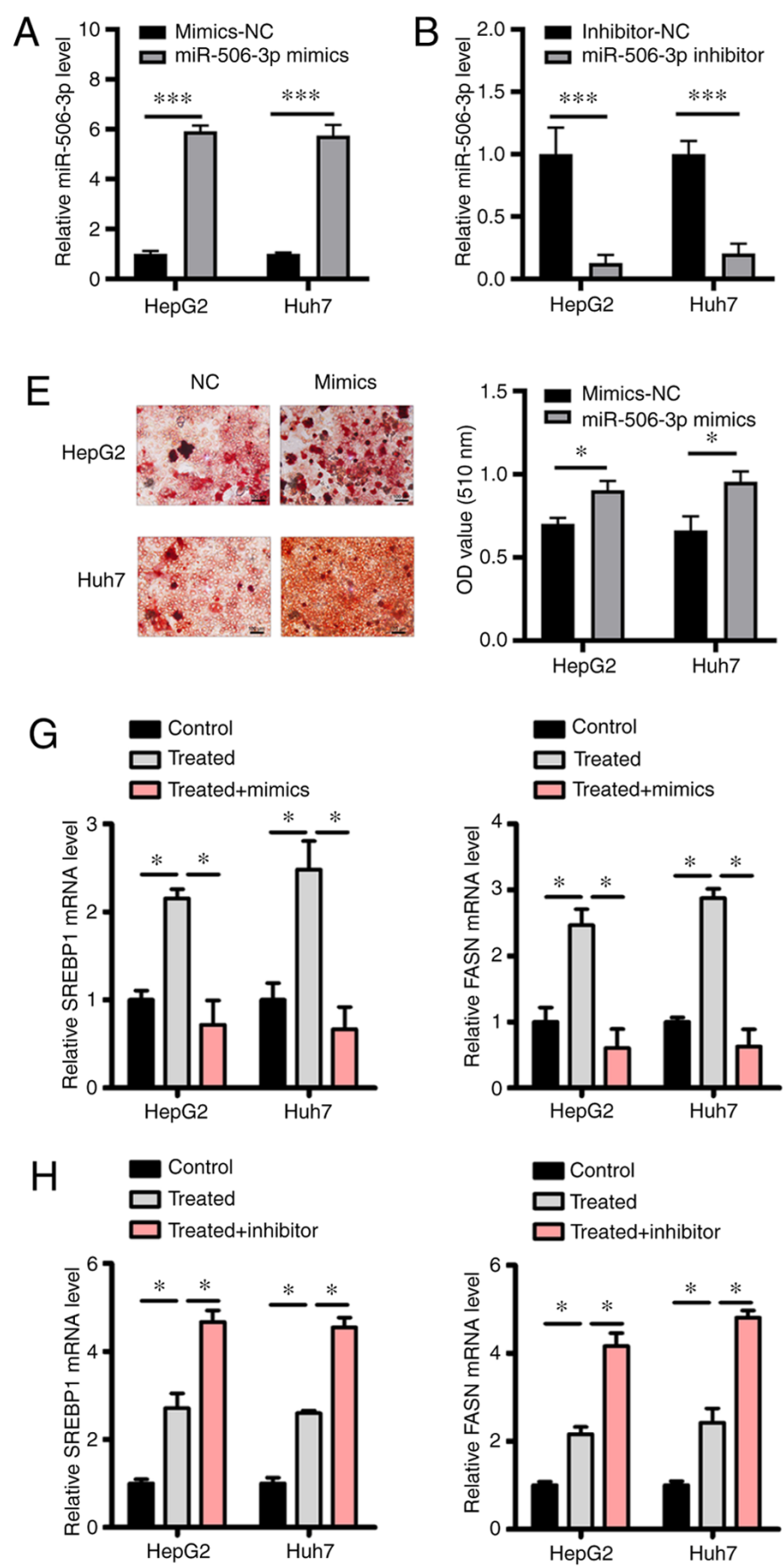
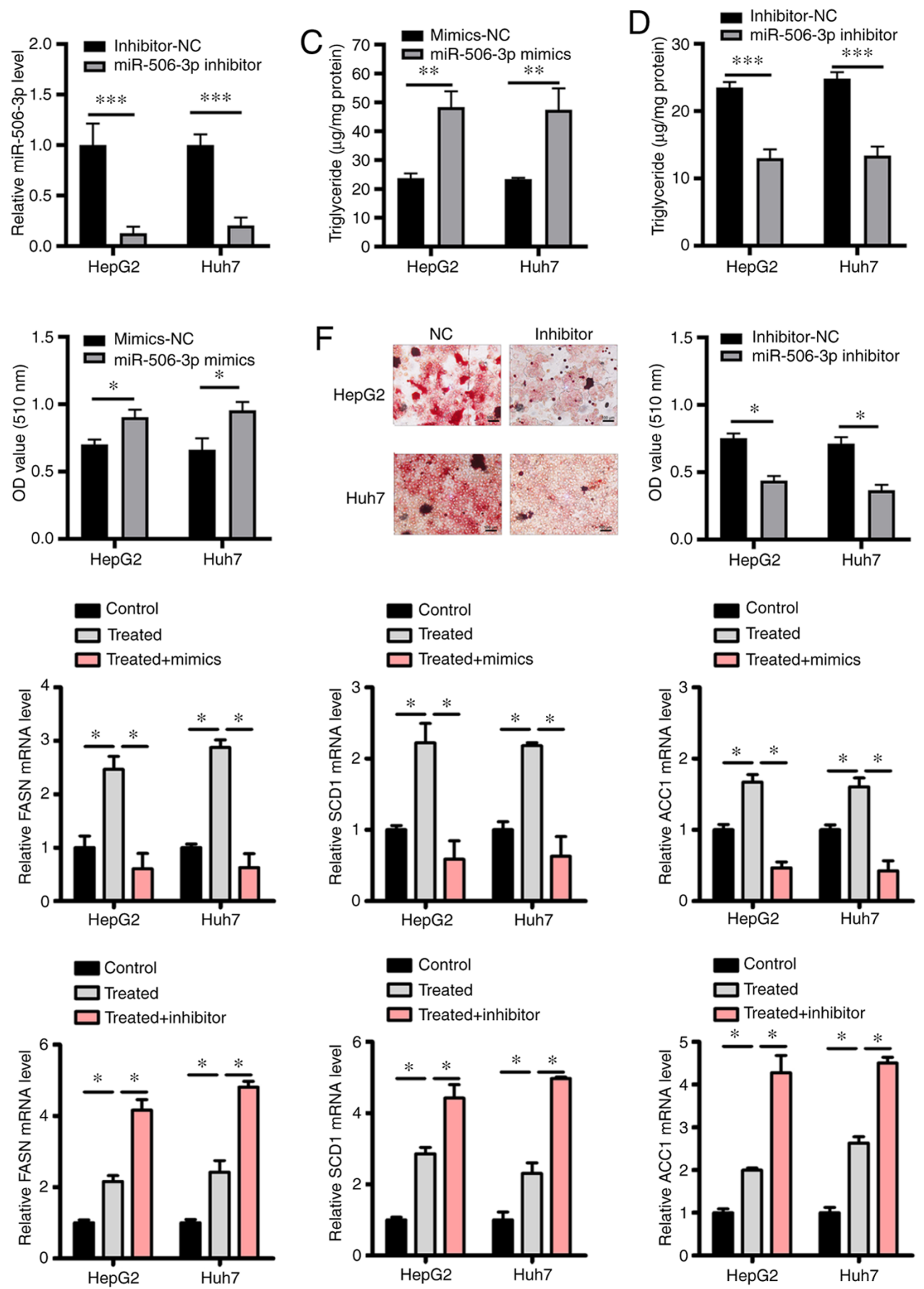
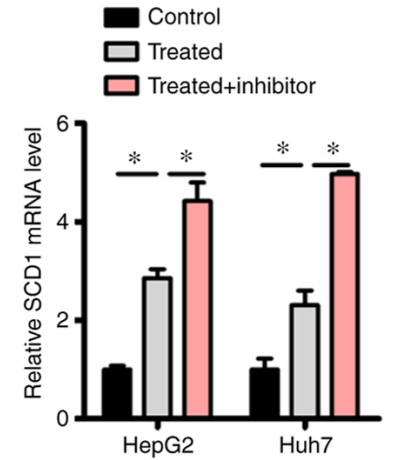

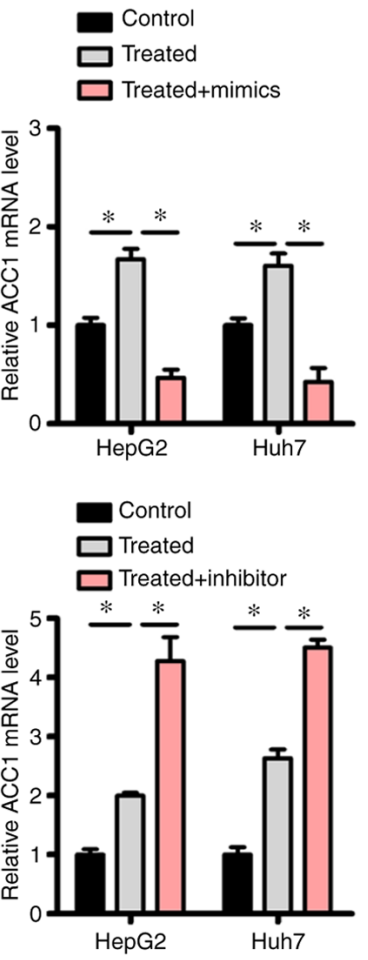

Figure 2. Overexpression of miR-506-3p induces lipid accumulation in HepG2 and Huh7 cells. (A and B) Following transfection with miR-506-3p mimics or inhibitor, miR-506-3p expression in HepG2 and Huh7 cells was quantified via RT-qPCR. (C and D) HepG2 and Huh7 cells were treated with high glucose prior to transfection with miR-506-3p mimics or inhibitor or with corresponding NC constructs, and an enzymatic method was used to measure intracellular triglyceride levels. (E and F) HepG2 and Huh7 cells were treated with high glucose prior to transfection with miR-506-3p mimics or inhibitor or with corresponding $\mathrm{NC}$ constructs. Oil Red O staining and quantification via spectrophotometry are presented. Scale bars, $100 \mu \mathrm{m}$. (G and $\mathrm{H})$ The relative expression levels of SREBP1, FASN, SCD1 and ACC1 in high-glucose treated HepG2 and Huh7 cells were detected by RT-qPCR. Data are presented as the mean \pm SD (n=3). ${ }^{*} \mathrm{P}<0.05 ;{ }^{* * *} \mathrm{P}<0.01 ;{ }^{* * *} \mathrm{P}<0.001$. miR, microRNA; SIRT1, sirtuin 1; NC, negative control; RT-qPCR, reverse transcription quantitative-PCR; SREBP1, sterol regulatory element-binding protein 1; FASN, fatty acid synthase; SCD1, stearoyl-CoA desaturase-1; ACC1, acetyl-CoA carboxylase 1; OD, optical density.

increased in glucose-treated cells compared with control cells (Fig. 1B). miR-506-3p and SIRT1 expression level was examined in these cells, revealing that miR-506-3p expression level was significantly increased in glucose-treated cells (Fig. 1C), whereas SIRT1 expression was markedly decreased at the RNA and protein level in both cell lines following high glucose treatment (Fig. 1D-F).
miR-506-3p induces lipid accumulation in HepG2 and Huh7 cells. To explore the functional relevance of miR-506-3p in the context of hepatic steatosis, HepG2 and Huh7 cells were transfected with miR-506-3p mimics or inhibitor (Fig. 2A and B). The analysis of TG levels in these cells following high glucose pretreatment revealed that transfection with miR-506-3p mimics led to a significant enhancement of glucose-induced 

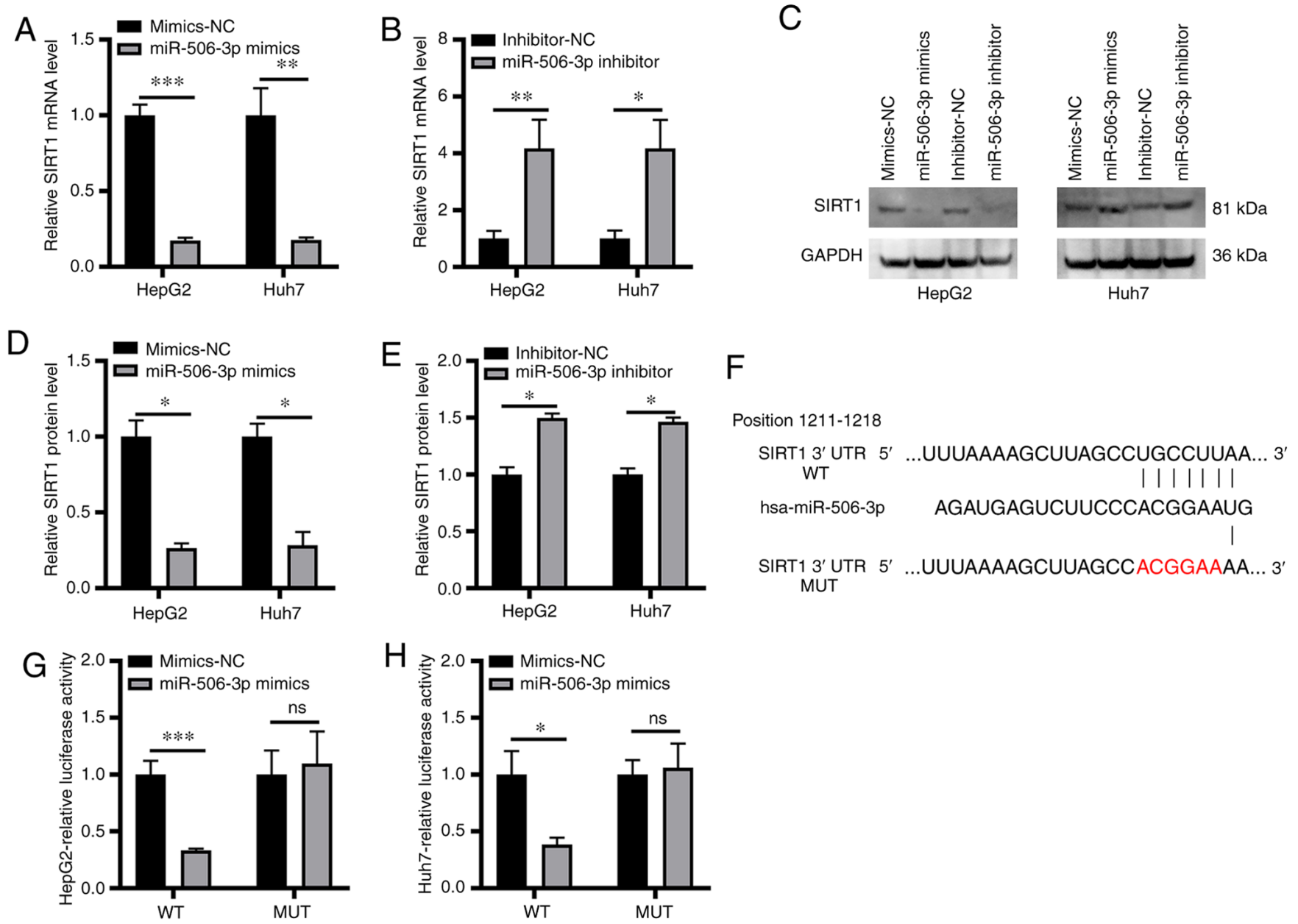

Figure 3. miR-506-3p binds to the 3'-UTR of SIRT1 and suppresses its expression. HepG2 and Huh7 cells were transfected with miR-506-3p mimics or inhibitor, and SIRT1 levels were quantified via (A and B) reverse transcription quantitative-PCR and (C-E) western blotting. (F) Putative miR-506-3p binding site in the SIRT1 3'-UTR, with the mutant sequence additionally presented in red font. (G and H) The interaction between miR-506-3p and SIRT1 3'-UTR was confirmed via dual luciferase reporter assay. Data are presented as the mean $\pm \mathrm{SD}(\mathrm{n}=3) .{ }^{*} \mathrm{P}<0.05 ;{ }^{* *} \mathrm{P}<0.01 ;{ }^{* * *} \mathrm{P}<0.001$. miR, microRNA; SIRT1, sirtuin $1 ; \mathrm{NC}$, negative control; UTR, untranslated region; WT, wild-type; MUT, mutant; ns, not significant.

TG accumulation compared with the control group, whereas miR-506-3p inhibitor transfection exhibited the opposite effect (Fig. 2C and D). miR-506-3p mimics also resulted in significant lipid accumulation within these cells compared with the control group, demonstrated via Oil Red O staining, whereas miR-506-3p inhibitor transfection had the opposite effect (Fig. 2E and F). Subsequently, the expression levels of major lipogenic genes, such as sterol regulatory elementbinding protein 1 (SREBP1), fatty acid synthase (FASN), stearoyl-CoA desaturase-1 (SCD1) and acetyl-CoA carboxylase 1 (ACC1) were examined $(21,22)$ in cells treated with high glucose concentration with or without transfection with miR-506-3p inhibitor or mimics. Treatment with high glucose increased the expression level of these genes, as indicated by RT-qPCR; however, their expression levels were decreased by miR-506-3p mimics (Fig. 3G) and increased by miR-506-3p inhibitor compared with non-transfected cells treated with high-glucose (Fig. 3H).

miR-506-3p binds to the 3'-UTR of SIRT1 and suppresses its expression. The association between SIRT1 and miR-506-3p in the cellular model of hepatic steatosis was further explored.
miR-506-3p mimics transfection in cells resulted in a significant reduction in SIRT1 expression at the mRNA and protein level, whereas the opposite effect was observed following miR-506-3p inhibitor transfection (Fig. 3A-E). These data suggested that miR-506-3p may directly or indirectly regulate SIRT1 in this molecular context. To further explore this regulatory association, the potential complementarity between SIRT1 mRNA and miR-506-3p was examined, revealing a putative miR-506-3p binding site in the SIRT1 3'-UTR (Fig. 3F). Luciferase reporter assay revealed that miR-506-3p mimics significantly suppressed the luciferase activity of reporter constructs containing the wild-type but not a mutated version of the SIRT1 3'-UTR sequence (Fig. 3G and H). These results suggested that miR-506-3p could directly bind to the 3'-UTR of SIRT1 and suppress its expression.

miR-506-3p modulates AMPK phosphorylation in an in vitro model of hepatic steatosis. The AMPK pathway has been indicated to be associated with the onset of NAFLD (23), and enhance intracellular NAD ${ }^{+}$levels, thereby leading to enhanced SIRT1 activation (24). Similarly, SIRT1 overexpression has been demonstrated to result in increased AMPK activation, 


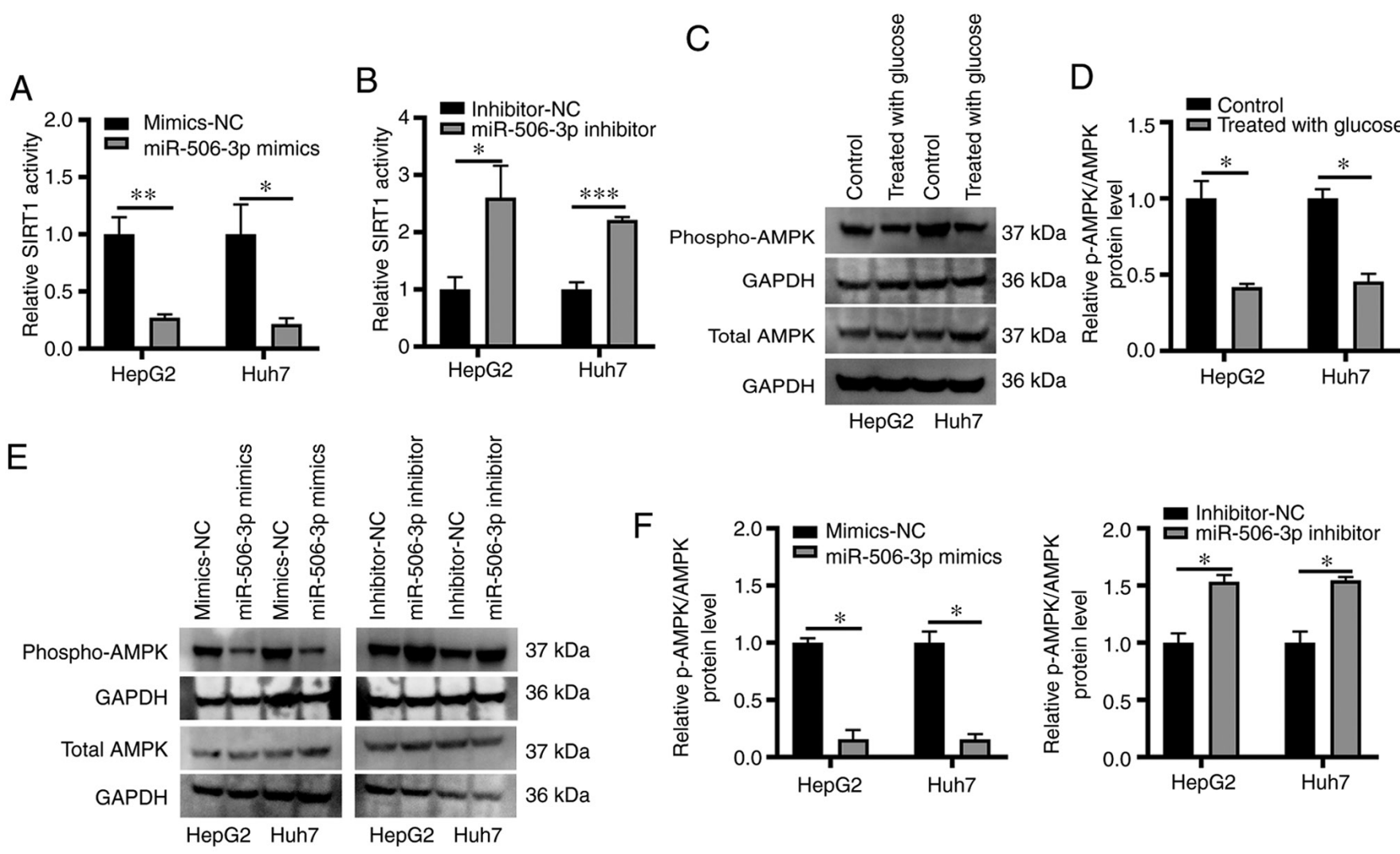

Figure 4. miR-506-3p modulates AMPK phosphorylation in an in vitro model of hepatic steatosis. (A and B) A fluorometric assay was used to measure SIRT1 activity in HepG2 and Huh7 cells without high glucose treatment transfected with miR-506-3p mimics or inhibitor. (C and D) AMPK phosphorylation was examined via western blotting in HepG2 and Huh7 cells treated with high glucose, and densitometry was used for quantification purposes. (E and F) Western blotting was performed to observe the effect of miR-506-3p mimics or inhibitor transfection on AMPK phosphorylation in HepG2 and Huh7 cells not treated with high glucose. Data are presented as the mean $\pm \mathrm{SD}(\mathrm{n}=3) .{ }^{*} \mathrm{P}<0.05 ;{ }^{* *} \mathrm{P}<0.01 ;{ }^{* * *} \mathrm{P}<0.001$. miR, microRNA; AMPK, AMP-activated protein kinase, SIRT1, sirtuin 1; NC, negative control; p, phosphorylated.

highlighting a regulatory feedback mechanism in this metabolic context (25). In the present study, SIRT1 deacetylase activity was measured in samples obtained from HepG2 and Huh7 cells that were not treated with high glucose transfected with miR-506-3p mimics or inhibitor using a fluorometric assay, revealing that miR-506-3p mimics transfection resulted in markedly reduced SIRT1 activity, whereas miR-506-3p inhibition exhibited the opposite effect (Fig. 4A and B). The role of the AMPK pathway as a mediator of SIRT1 activity in this cellular model of hepatic steatosis was subsequently examined by detecting AMPK phosphorylation via western blotting. The results revealed that lipid accumulation in high glucose-treated HepG2 and Huh7 cells was associated with a significant reduction in AMPK phosphorylation (Fig. 4C and $\mathrm{D}$ ). In addition, miR-506-3p mimics transfection into these cells significantly decreased the level of AMPK phosphorylation, whereas miR-506-3p inhibitor transfection led to significantly enhanced AMPK phosphorylation compared with the control group (Fig. 4E and F).

\section{Discussion}

NAFLD is a disease that arises from hepatic steatosis, which is itself associated with other metabolic disorders, including insulin resistance, hyperglycemia and elevated fatty acid levels (26). Consistent with these associations, the present study indicated that treatment of Huh7 and HepG2 cells with high glucose levels led to increased uptake of lipids by these hepatocytes, thus generating an in vitro model of hepatic steatosis.

SIRT1 is a key regulator of lipid metabolism within cells that improves tissue insulin sensitivity and suppresses the onset of hepatic steatosis (27). Significant SIRT1 downregulation was observed in HepG2 and Huh7 cells following high glucose treatment and lipid accumulation. A previous study has demonstrated that SIRT1 activity could prevent the onset of hepatic steatosis, and that patients with NAFLD present lower SIRT1 expression compared with healthy individuals (3). Knockdown of SIRT1 in hepatocytes has been indicated to result in decreased fatty acid oxidation, whereas lipid metabolism was enhanced via peroxisome proliferator-activated receptor- $\alpha$ signaling upon SIRT1 overexpression (28). Consistent with these findings, mice with a hepatocyte-specific SIRT1 knockout exhibited increased steatosis and inflammation (28). However, the mechanistic basis of SIRT1 effect on hepatic steatosis remains poorly elucidated.

A previous study has highlighted the ability of miRNAs to modulate a wide range of physiological and pathological conditions owing to their ability to post-transcriptionally regulate mRNA translation and degradation $(29,30)$. The present study indicated SIRT1 to be a predicted target of miR506-3p, with a notable complementarity between miR-506-3p 
and SIRT1 3'-UTR. A significant increase in miR-506-3p expression level was observed in high glucose-treated HepG2 and Huh7 cells that coincided with intracellular lipid accumulation. Interestingly, transfection of these hepatocytes with miR-506-3p mimics resulted in a significant enhancement in lipid accumulation within these cells upon glucose exposure, whilst transfection of these hepatocytes with miR-506-3p inhibitor resulted in a significant decrease in lipid accumulation within these cells upon glucose exposure. This suggests that miR-506-3p serves a direct role in regulating the development of hepatic steatosis.

To explore the mechanisms by which miR-506-3p regulated the present model of hepatic steatosis, SIRT1 expression was examined in particular, owing to SIRT1 downregulation in NAFLD and its high complementarity with miR-506-3p (31). Previous studies have suggested that SIRT1 upregulation may be of therapeutic value in NAFLD (32), resulting in altered regulation of a number of key metabolism-related genes, including SREBP1, FASN and SCD1 $(33,34)$. Using a luciferase reporter assay, a direct interaction between miR-506-3p and SIRT1 3'-UTR was confirmed. Multiple miRNAs have previously been demonstrated to promote lipogenesis and therefore drive the onset of NAFLD, including miR-9 (35) and miR-34a (36). The present study, however, is the first to similarly highlight miR-506-3p as a potential regulator of NAFLD owing to its ability to inhibit SIRT1 expression, to the best of our knowledge.

AMPK activation has been indicated to decrease lipid production and enhance fatty acid oxidation, ultimately inhibiting hepatic TG accumulation and NAFLD development (37). In the present study, a significant reduction in AMPK phosphorylation level following high glucose treatment in HepG2 and Huh7 cells was observed, while miR-506-3p inhibitor transfection significantly enhanced AMPK phosphorylation and miR-506-3p mimic transfection significantly decreased AMPK phosphorylation. These data suggested that the SIRT1/ AMPK axis mediated at least partially the metabolic effects of miR-506-3p in the present in vitro model of hepatic steatosis.

In conclusion, the results of the present study suggested that miR-506-3p suppressed SIRT1 expression, thereby impairing AMPK signaling. These results further suggested that miR506-3p may be associated with the pathogenesis of hepatic steatosis and may represent either a biomarker or a therapeutic target in NAFLD.

\section{Acknowledgements}

Not applicable.

\section{Funding}

The present study was supported by Shanghai Science and Technology Committee (grant no. 17ZR1427000), Shanghai Municipal Health Committee (grant no. 201740156), National Natural Science Foundation of China (grant no. 81772591), National Key Basic Research Program of China (grant no. 2014CB542102), Shanghai Health and Family Planning Commission Foundation (grant no. 20164Y0189), National Human Genetic Resources Sharing Service Platform (grant no. 2005DKA21300), Science Fund for Creative Research
Groups, NSFC (grant no. 81521091) and State Key Infectious Disease Project of China (grant no. 2017ZX10203208).

\section{Availability of data and materials}

The datasets used and/or analyzed during the current study are available from the corresponding author on reasonable request.

\section{Authors' contributions}

XFX conceived the study and designed the experiments. LKH, JQC, HZ, YPT, YY and XFX performed the experimnts. LKH, JQC, HZ, YPT and XFX wrote and revised the manuscript. All authors contributed to the interpretation and discussion of the results and reviewed the manuscript. LKH, JQC and XFX confirm the authenticity of all the raw data. All authors have read and approved the final manuscript.

\section{Ethics approval and consent to participate}

Not applicable.

\section{Patient consent for publication}

Not applicable.

\section{Competing interests}

All authors declare that they have no competing interests.

\section{References}

1. Gui Y, Pan Q, Chen X, Xu S, Luo X and Chen L: The association between obesity related adipokines and risk of breast cancer: A meta-analysis. Oncotarget 8: 75389-75399, 2017.

2. Benedict $\mathbf{M}$ and Zhang X: Non-alcoholic fatty liver disease: An expanded review. World J Hepatol 9: 715-732, 2017.

3. Nassir F and Ibdah JA: Sirtuins and nonalcoholic fatty liver disease. World J Gastroenterol 22: 10084-10092, 2016.

4. Tobita T, Guzman-Lepe J, Takeishi K, Nakao T, Wang Y, Meng F, Deng CX, Collin de l'Hortet A and Soto-Gutierrez A: SIRT1 disruption in human fetal hepatocytes leads to increased accumulation of glucose and lipids. PLoS One 11: e0149344, 2016.

5. Arab SadeghabadiZ,Nourbakhsh M,Pasalar P,EmamgholipourS, Golestani A, Larijani B and Razzaghy-Azar M: Reduced gene expression of sirtuins and active AMPK levels in children and adolescents with obesity and insulin resistance. Obes Res Clin Pract 12: 167-173, 2018.

6. Kitada M and Koya D: SIRT1 in type 2 diabetes: mechanisms and therapeutic potential. Diabetes Metab J 37: 315-325, 2013.

7. Hardie DG: Minireview: the AMP-activated protein kinase cascade: the key sensor of cellular energy status. Endocrinology 144: 5179-5183, 2003

8. Hou X, Xu S, Maitland-Toolan KA, Sato K, Jiang B, Ido Y, Lan F, Walsh K, Wierzbicki M, Verbeuren TJ, et al: SIRT1 regulates hepatocyte lipid metabolism through activating AMP-activated protein kinase. J Biol Chem 283: 20015-20026, 2008.

9. Jinek $M$ and Doudna JA: A three-dimensional view of the molecular machinery of RNA interference. Nature 457: 405-412, 2009.

10. Krol J, Loedige I and Filipowicz W: The widespread regulation of microRNA biogenesis, function and decay. Nat Rev Genet 11: 597-610, 2010.

11. Rottiers V and Näär AM: MicroRNAs in metabolism and metabolic disorders. Nat Rev Mol Cell Biol 13: 239-250, 2012.

12. Ceccarelli S, Panera N, Gnani D and Nobili V: Dual role of microRNAs in NAFLD. Int J Mol Sci 14: 8437-8455, 2013. 
13. Kerr TA, Korenblat KM and Davidson NO: MicroRNAs and liver disease. Transl Res 157: 241-252, 2011.

14. Lakner AM, Bonkovsky HL and Schrum LW: microRNAs: Fad or future of liver disease. World J Gastroenterol 17: 2536-2542, 2011.

15. Hu CY, You P, Zhang J, Zhang H and Jiang N: MiR-506-3p acts as a novel tumor suppressor in prostate cancer through targeting GALNT4. Eur Rev Med Pharmacol Sci 23: 5133-5138, 2019.

16. Deng Q, Xie L and Li H: MiR-506 suppresses cell proliferation and tumor growth by targeting Rho-associated protein kinase 1 in hepatocellular carcinoma. Biochem Biophys Res Commun 467: 921-927, 2015.

17. Wang Z, Si M, Yang N, Zhang H, Fu Y, Yan K, Zong Y, Zhu N and Wei Y: MicroRNA-506 suppresses invasiveness and metastasis of human hepatocellular carcinoma cells by targeting IL8. Am J Cancer Res 8: 1586-1594, 2018.

18. Debes JD, Boonstra A and de Knegt RJ: NAFLD-related hepatocellular carcinoma and the four horsemen of the apocalypse. Hepatology 71: 774-776, 2020.

19. Teras LR, DeSantis CE, Cerhan JR, Morton LM, Jemal A and Flowers CR: 2016 US lymphoid malignancy statistics by World Health Organization subtypes. CA Cancer J Clin 66: 443-459, 2016.

20. Livak KJ and Schmittgen TD: Analysis of relative gene expression data using real-time quantitative PCR and the 2(-Delta Delta C(T)) Method. Methods 25: 402-408, 2001

21. Lin MJ, Dai W, Scott MJ, Li R, Zhang YQ, Yang Y, Chen LZ and Huang XS: Metformin improves nonalcoholic fatty liver disease in obese mice via down-regulation of apolipoprotein A5 as part of the AMPK/LXR $\alpha$ signaling pathway. Oncotarget 8: 108802-108809, 2017.

22. da Silva-Santi LG, Antunes MM, Caparroz-Assef SM, Carbonera F, Masi LN, Curi R, Visentainer JV and Bazotte RB: Liver fatty acid composition and inflammation in mice fed with high-carbohydrate diet or high-fat diet. Nutrients 8: 8, 2016.

23. Santamarina AB, Oliveira JL, Silva FP, Carnier J, Mennitti LV, Santana AA, de Souza GH, Ribeiro EB, Oller do Nascimento CM, Lira FS, et al: Green tea extract rich in epigallocatechin-3-gallate prevents fatty liver by AMPK activation via LKB1 in mice fed a high-fat diet. PLoS One 10: e0141227, 2015.

24. Cantó C, Gerhart-Hines Z, Feige JN, Lagouge M, Noriega L, Milne JC, Elliott PJ, Puigserver P and Auwerx J: AMPK regulates energy expenditure by modulating $\mathrm{NAD}^{+}$metabolism and SIRT 1 activity. Nature 458: 1056-1060, 2009.

25. Lan F, Cacicedo JM, Ruderman N and Ido Y: SIRT1 modulation of the acetylation status, cytosolic localization, and activity of LKB1. Possible role in AMP-activated protein kinase activation J Biol Chem 283: 27628-27635, 2008.

26. Kitade $\mathrm{H}$, Chen G, Ni Y and Ota T: Nonalcoholic fatty liver disease and insulin resistance: new insights and potential new treatments. Nutrients 9: 9, 2017.
27. Cao Y, Jiang X, Ma H, Wang Y, Xue P and Liu Y: SIRT1 and insulin resistance. J Diabetes Complications 30: 178-183, 2016.

28. Purushotham A, Schug TT, Xu Q, Surapureddi S, Guo X and Li X: Hepatocyte-specific deletion of SIRT1 alters fatty acid metabolism and results in hepatic steatosis and inflammation. Cell Metab 9: 327-338, 2009.

29. Yuan JH, Yang F, Wang F, Ma JZ, Guo YJ, Tao QF, Liu F, Pan W, Wang TT, Zhou CC, et al: A long noncoding RNA activated by TGF- $\beta$ promotes the invasion-metastasis cascade in hepatocellular carcinoma. Cancer Cell 25: 666-681, 2014.

30. Bartel DP: MicroRNAs: Genomics, biogenesis, mechanism, and function. Cell 116: 281-297, 2004.

31. Castro RE, Ferreira DM, Afonso MB, Borralho PM, Machado MV, Cortez-Pinto H and Rodrigues CM: miR-34a/ SIRT1/p53 is suppressed by ursodeoxycholic acid in the rat liver and activated by disease severity in human non-alcoholic fatty liver disease. J Hepatol 58: 119-125, 2013.

32. Colak Y, Yesil A, Mutlu HH, Caklili OT, Ulasoglu C, Senates E, Takir M, Kostek O, Yilmaz Y, Yilmaz Enc F, et al: A potential treatment of non-alcoholic fatty liver disease with SIRT1 activators. J Gastrointestin Liver Dis 23: 311-319, 2014.

33. Wang LF, Wang XN, Huang CC, Hu L, Xiao YF, Guan XH, Qian YS, Deng KY and Xin HB: Inhibition of NAMPT aggravates high fat diet-induced hepatic steatosis in mice through regulating Sirt1/AMPK $\alpha /$ SREBP1 signaling pathway. Lipids Health Dis 16: 82, 2017.

34. Sun L, Wang Y, Song Y, Cheng XR, Xia S, Rahman MR, Shi Y and Le G: Resveratrol restores the circadian rhythmic disorder of lipid metabolism induced by high-fat diet in mice. Biochem Biophys Res Commun 458: 86-91, 2015.

35. Ao R, Wang Y, Tong J and Wang BF: Altered microRNA-9 Expression Level is Directly Correlated with Pathogenesis of Nonalcoholic Fatty Liver Disease by Targeting Onecut 2 and SIRT1. Med Sci Monit 22: 3804-3819, 2016.

36. Kim HJ, Joe Y, Yu JK, Chen Y, Jeong SO, Mani N, Cho GJ, Pae HO, Ryter SW and Chung HT: Carbon monoxide protects against hepatic ischemia/reperfusion injury by modulating the miR-34a/SIRT1 pathway. Biochim Biophys Acta 1852: $1550-1559,2015$

37. Boudaba N, Marion A, Huet C, Pierre R, Viollet B and Foretz M: AMPK re-activation suppresses hepatic steatosis but its downregulation does not promote fatty liver development. EBioMedicine 28: 194-209, 2018.

(i) $\odot$ This work is licensed under a Creative Commons Attribution-NonCommercial-NoDerivatives 4.0 International (CC BY-NC-ND 4.0) License. 